\title{
Bettina Lindorfer
}

\section{Der Diskurs der Geschichte und der Ort des Realen}

\author{
Roland Barthes' Beitrag zum linguistic turn \\ der Geschichtsschreibung
}

\section{Geschichte und Sprache: der linguistic turn}

Die vielbeschworene Krise der Geschichtsschreibung ist auch eine Krise ihres Wirklichkeitsbegriffs. Roger Chartier führt die große Mutation, die die Geschichtswissenschaft seit Mitte der 80er Jahre erfaßt hat, auf einen zweifachen Perspektivenwechsel zurück. Einerseits habe der Einzug des Individuums die Mikrogeschichte und die Individuen fokussierende Sozialgeschichte entstehen lassen ${ }^{1}$; andererseits habe die Erkenntnis, daß jeder geschichtliche Diskurs letztlich eine Erzählung ( den Vordergrund gerückt. Der linguistic turn der Geschichtsschreibung ist für Chartier die schlechte Radikalisierung dieser Erkenntnis. Ausgehend von Saussures Konzeption der Sprache ${ }^{2}$ als einem geschlossenen System von Zeichen habe diese Wende zu einem Blick auf Texte geführt, der sich von der Geschichte als Sozialwissenschaft verabschiedet habe. Denn die Zeichen erhielten hier ihre Bedeutung (signification) nicht über den Gebrauch und den sozialen Kontext, sondern aus ihrer Beziehung zu anderen Zeichen. "La production du sens est ainsi détachée de toute intention ou de tout contrôle subjectifs puisqu'elle se trouve assignée à un fonctionnement linguistique automatique et impersonel. La réalité n'est plus à penser comme une référence objective, extérieure au discours, mais comme constituée par et dans le langage. ${ }^{3}$

Es ist Derridas in der Grammatologie ausgegebene Parole "il n'y a pas de horstexte", die die Macht der Sprache und des Textes sowie den "Verlust des Subjekts

\footnotetext{
1 Chartier (1995) 34-37.
}

2 Le langage: Sprache und Sprechen', sagt Chartier, während Saussure natürlich nur die langue: ,das Sprachsystem', meint. Diese scheinbar minimale Differenz führt zu einer denkwürdigen rencontre manquée von Sprachwissenschaft und Geschichte - z. B. auch bei Iggers (1995) $558 \mathrm{ff} ., 568 \mathrm{ff}$.

3 Chartier (1995) 41; vgl. Hanisch (1996) 215. 
und der Welt" begründet ${ }^{4}$. Sie führt die Geschichtswissenschaft natürlich nicht dazu, zu Sprachanalysen überzugehen, aber sehr wohl dazu, die Textualität ihrer Quellen nun stärker unter die Lupe zu nehmen. Die Frage nach deren Tatsachenbezogenheit tritt dabei manchmal in den Hintergrund; zumindest ist sie nicht immer das allein entscheidende Kriterium. So unternehmen es Dominick LaCapra und Hayden White, Gerichtsakten als Texte zu lesen, die ihrerseits hauptsächlich auf andere Texte verweisen; die Frage nach ihrer Glaubwürdigkeit bleibt dabei abgeblendet.

Allerdings wird eine solche Literarisierung der historischen Quellen keineswegs einhellig begrüßt. Eingewendet wurde, daß in der Fokussierung auf konkrete Details sehr wohl die sozialen und kulturellen Bedeutungssysteme der Erzählenden erkennbar werden. Nach Carlo Ginzburg liefern sie die „Spuren“ vergangener Ereignisse, die es zu sichern und im Hinblick auf historische Fakten zu dechiffrieren gelte.

Nathalie Zemon Davis' Untersuchung zu Gnadenbriefen an den französischen König im 16. Jahrhundert nahm hierzu eine Kompromißposition ein. Der programmatische Titel "Fiction in the archives“ weist bereits darauf hin, daß Zemon Davis diese Bittbriefe nicht primär als eine Quelle auswertet, die über die tatsächlichen Handlungssequenzen eines Verbrechens Auskunft gibt, sondern zuallererst als Literatur. Die Historikerin liest die lettres de rémission als Texte, die juristische, historische und narrative Informationen mischten, die aber gerade nicht, wie die traditionelle Historiographie dies fordere, primär auf ihren „realen Kern“ zu befragen seien. Sie macht also keinen prinzipiellen Unterschied zwischen juristischem Gnadengesuch und historischem Bericht der Petenten über die eigene Tat einerseits und den ,fiktiven' Elementen der Bittbriefe andererseits. Zemon Davis will herausfinden, „wie Menschen im 16. Jahrhundert Geschichten erzählten“. "Wie wahrheitsgetreu“ sie dies in ihren Gnadengesuchen tun, wird erst in zweiter Instanz untersucht. Damit erhalten textuelle bzw. ,fiktionale' Aspekte, d.h. „die Elemente der Quellen, die eine Geschichte hervorbringen, formen und gestalten ${ }^{4}$, einen symptomatischen Stellenwert, auch wenn sie zuletzt doch noch an eruierbare ,Fakten' zurückgebunden werden.

Die Gleichwertigkeit narrativer und ,harter' Quellen bei Zemon Davis hat Schule gemacht ${ }^{6}$. Auch für Peter Burke ist es weder allein maßgebliches Kriterium noch endgültig entscheidbar, ob die Reiseberichte aus dem Italien des 15. Jahrhunderts, historische Tatsachen“ wiedergeben. Der Historiker solle sich gerade nicht in einen falschen Gegensatz zwischen literarischer ,Fiktion' und belegbaren ,Tatsachen' zwängen lassen. „Natürlich“ könne man den zeitgenössischen Autoren „nicht ... jedes Wort" glauben, weil sie ungenau, verzerrt oder nur vom Hörensagen berichtet oder Beobachtetes falsch interpretiert haben können. Noch wenn

+ Vgl. Daniel (1997) $262 \mathrm{f}$.

5 Zemon Davis (1988) 16.

6 Siehe z. B. Dinges (1994) zu Beleidigungställen im Paris des 18. oder Minkmar (1996) zu Colmar im 16. Jahrhundert und allgemein Schwerboff (1999). 
ihre Aufzeichnungen in Einzelheiten mit den Berichten anderer Zeitgenossen übereinstimmten, sei nicht auszuschließen, daß dann einfach Gemeinplätze wiederholt oder von anderen Berichten abgeschrieben worden seien ${ }^{7}$. Deshalb stellten zeitgenössische Quellen lediglich „Zeugnisse einer kulturell stereotypisierten Wahrnehmung“ dar, bei denen Burke allerdings annimmt, daß eine „komplexe Beziehung“ zu "der sozialen Realität, die ihnen zugrunde liegt ${ }^{\text {“ }}$ (85), bestehe.

Angesichts solcher Formulierungen verwundert es kaum, wenn in der Geschichtsschreibung längst die Forderung nach einem Jenseits des linguistic turn gestellt worden ist. Statt sich mit Diskursen auseinanderzusetzen, mit Sprache und Zeichen, „die die Wahrnehmung der Welt strukturieren “8, ist es z. B. für Lyndal Roper an der Zeit, endlich das Verhältnis neu zu bestimmen zwischen dem „Diskurs" und dem, „was in der Geschichtswissenschaft einst als Realität bezeichnet wurde“. Roper will gerade die Dinge erforschen, „die sich dem sprachlichen Ausdruck entziehen" (453): die Subjektivität individueller Erfahrung, „die Art und Weise, wie jemand mental und emotional seine Erfahrung strukturiert" (457), ndie Dimension des Unbewußten“ (458), die Phantasien und andere für Handlungen bestimmende Triebkräfte (462). Die Historiographie müsse über die textuellen Spuren historischer Quellen hinausgehen, um "die Phantasie als historische Kraft zu betrachten und das Unbewußte als Bestandteil der Geschichte zu verstehen“" (465).

Die Geschichtsschreibung ist aber nicht nur in ihren Quellen sprachgebunden. Nicht nur stellen sprachliche Dokumente (Gesetzestexte, Gerichtsprotokolle, Erntestatistiken etc.) ihren primären Forschungsgegenstand dar, auch sie selbst ereignet sich sozusagen sprachlich; sie produziert Texte, in denen sie darstellt, erzählt, wie etwas gewesen ist. Während die Problematisierung der Quellen in bezug auf Wahrheitswert und Realitätsabbildung doch eine gewisse Tradition hat, scheint dieser zweite Aspekt des linguistic turn Neues in die Betrachtung der Geschichte eingebracht zu haben. Von der Begrifflichkeit über die Themenwahl bis zu Erkenntnisinteresse und Deutungskonstruktionen beleuchtet er nun historiographische Vertextungsstrategien. Vor allem die Werke großer Historiker des 19. Jahrhunderts rücken als literarische Artefakte in den Blick. Ob nun Roland Barthes die antithetischen Obsessionen eines Michelet ${ }^{9}$ hervortreten läßt oder Hayden White Marx, Nietzsche oder Ranke auf einen metaphorischen, metonymischen oder synekdochischen Erzählstil ${ }^{10}$ festlegt: Plötzlich werden diese Arbeiten vorrangig in ihrer Darstellungsweise und weniger als Darstellung von historischen Ereignissen betrachtet. - Dies wäre der oft beschworene „postmoderne Perspektivenwechsel"11: Die Disziplin befragt ihre Darstellungsformen. Zum Beispiel fragt sie, was den historiographischen vom literarischen Text trennt. Die Frage ist allerdings nicht ganz neu. So schrieb bereits Aristoteles im Kapitel 9 der

7 Vgl. Burke (1996) 85, 26, 90.

8 Roper (1999) 452.

9 Barthes, Michelet (1964), in: Euvres complètes, Bd. I.

10 White (1994); vgl. Daniel (1997) 273.

"Daniel (1997) 262. 
Poetik: "Der Geschichtsschreiber und der Dichter unterscheiden sich nicht dadurch voneinander, daß sich der eine in Versen und der andere in Prosa mitteilt ...; sie unterscheiden sich vielmehr dadurch, daß der eine das wirklich Geschehene mitteilt, und der andere, was geschehen könnte. ${ }^{* 12}$

Unterscheiden sich die Werke von Dichtern und Geschichtsschreibern also nicht in ihrer Textualität? Sieht man ihnen selbst nicht an, ob sie, wirklich Geschehenes' mitteilen oder bloß Mögliches und Wahrscheinliches? Aristoteles scheint die textimmanente Beantwortung dieser Frage von sich zu weisen, wenn er das Kriterium Vers oder Prosa als nicht pertinent kennzeichnet. Er gibt aber doch einen Hinweis auf textuelle Indizien für Wahrheit und Realitätsgehalt, wenn er die Dichtung „etwas Philosophischeres und Ernsthafteres“ mitteilen sieht als die Geschichtsschreibung und dies damit begründet, daß die Dichtung ${ }_{n}$ mehr das Allgemeine, die Geschichtsschreibung hingegen das Besondere" mitteile (ebd.). Möglicherweise läßt sich schon aus dieser Bemerkung des Aristoteles eine Detailfixiertheit der Historiographie ableiten, die, wie mit Aristoteles zu ergänzen wäre, nicht nach den Regeln der Wahrscheinlichkeit oder Notwendigkeit vorgeht, sondern nach Maßgabe des wirklich Geschehenen. So nennen Geschichtsschreiber die Namen der Handelnden, während in der Dichtung höchstens die Namen herausragender Persönlichkeiten vorkommen ${ }^{13}$. Die Geschichte geht ins Detail.

Bevor solche ,unphilosophischen' Angaben näher betrachtet werden, möchte ich den Vorgang genauer untersuchen, der aus ihnen von Freud bis Morelli einen Index des Faktischen macht, und sie so mit der Kategorie des Wirklichen aufs innigste verquickt.

\section{Der Diskurs der Geschichte}

Dazu wende ich mich einem französischen Anfang des linguistic turn zu. Roland Barthes' Aufsatz „Le discours de l'histoire“ (1967) ist einer der ersten, der die unhintergehbare Sprachlichkeit der Geschichtsschreibung (l'Histoire) in den Vordergrund stellt. Barthes versteht unter ${ }_{n}$ Histoire" zwar explizit die erzählende Geschichtsschreibung des 19. Jahrhunderts; gleichzeitig gilt die ihm aber als symptomatisch für Historiographie überhaupt ${ }^{14}$. Der zentrale Vorwurf, den Barthes ihr macht, betrifft sowohl ihr Verhältnis zum geschichtlichen Ereignis als auch zu ihrer eigenen Sprache. In der Art und Weise, wie Barthes diesen Vorwurf begrün-

12 Aristoteles (1982) 29.

$13 \mathrm{Vgl}$. dazu die Anmerkungen von Fuhrmann zum 9. Kapitel der Poetik in Aristoteles (1982) $113 \mathrm{ff}$.

14 So behauptet Barthes in seinem "Compte rendu d'enseignement" zu diesem Thema (Studienjahr 1966/67 an der École Pratique des Hautes Études), daß der im Aufsatz explizit nur dem 19. Jahrhundert zugeschlagene effet de réel ein Kennzeichen jeder Geschichtsschreibung sei. Der "signifié ultime de la narration historique" sei immer die "constitution du ,réel“" Der effet de réel wird hier als das Kriterium schlechthin ausgegeben ("le trait pertinent"), das sie vom fiktiven Erzählen unterscheidet (II, 452). 
det, spielen Begriffe und Denkfiguren der strukturalen Semiologie eine entscheidende Rolle. Die Geschichte hänge zwei Illusionen an (die, wie gleich zu sehen sein wird, einander bedingen), wenn sie erstens an der Konzeption von Dingen und Ereignissen festhalte, die von der Sprache unberührt existierten, und wenn sie zweitens die Sprache als ein bloßes Instrument der Wiedergabe begreife, in dem die Dinge bloß ausgedrückt (und nicht bedeutet) werden ${ }^{15}$. Am Ende des Aufsatzes wird Barthes eine Position vertreten, die den Dingen nur eine sprachliche Daseinsweise zuerkennt und die als „Weltlosigkeit“ der Barthesschen Sprachauffassung verstanden worden ist ${ }^{16}$. Demgegenüber werde ich mit Bezug auf das gesamte Werk zu zeigen versuchen, daß Barthes' Haltung gegenüber dem Wirklichen ambivalenter ist, als es in diesem Aufsatz scheint, und daß der Schriftsteller Barthes der versprachlichten Welt durchaus eine welthaltige Sprache an die Seite ste.len will. Seine Reflexionen über eine, notierende oder gar ,zeigende 'Schreibweise, bei der die Beziehung zwischen Fotografie und Text eine entscheidende Rolle spielt, werden dafür die entscheidenden Argumente liefern.

Der Aufsatz von 1967 behandelt die Diskursstrategien historiographischer Texte in den drei Abschnitten, 1. énonciation, 2. énoncé und 3. signification. Zunächst wird gezeigt, welche „Verschieber" den Äußerungsakt (énonciation) mit dem Geäußerten (énoncé) verknüpfen. Hier werden Shifter des Hörens (Quellenangaben) von solchen der Diskursorganisation (der zeitlichen Abfolge), des (fehlenden) Adressaten und der verschiedenen Rollen, in die der Historiker schlüpfen kann, unterschieden ${ }^{17}$. Der zweite Abschnitt weist den Modus und den Implizitheitsgrad des énoncé als Indikatoren der Art von Geschichtsschreibung aus, die, so Barthes, entweder metaphorisch oder metonymisch, bisweilen auch reflexiv sein könne ${ }^{18}$. Der (scheinbar paradoxerweise) signification genannte letzte Abschnitt erläutert schließlich die textuellen Strategien, die in der Geschichte Bedeuturgszuschreibungen möglichst gering halten sollen (Wir werden sehen, daß mit "signification“ nicht die Bedeutung von Wörtern und Ausdrücken, sondern der Sirn von Texten gemeint ist.). Angekündigt wird dieses Programm im ersten Satz des Abschnitts, der lautet: „Pour que l'Histoire ne signifie pas, il faut que ... ${ }^{\star}$. Im Zentrum stehen hier unscheinbare Details, als deren wahre Funktion nicht inhalt-

15 ,Le référent est détaché du discours, il lui devient extérieur, fondateur" und: „c'est le sigrifié lui-même qui est repoussé, confondu dans le référent" (Barthes, Le discours de l'histoire II, 425).

16 Trabant (2003).

17 Z. B. könne der Historiker nach Fustel de Coulanges' Motto von der, Keuschheit der Geschichte' den objektiven Chronisten mimen, der in den Augen Barthes' natürlich nur „une forme particulière d'imaginaire" darstellt: "ici l'historien prétend laisser le référent parler tou: seul“ (II, 420).

18 Damit ergibt sich eine überraschende Übereinstimmung zu Whites vier narrativen Grundmustern der Geschichtsschreibung des 19. Jahrhunderts. Neben Metapher und Metonymie treten hier Synekdoche (die Barthes gewiß auf die Seite der Metonymie schlagen würde) und Ironie - sicher die Figur, die der reflexiven Darstellung am nächsten kommt auf. 
liche Information, sondern die Authentifizierung der Darstellung herausgestellt wird.

Daß die narrative Historiographie des 19. Jahrhunderts der Vergangenheit angehört, ist klar. Die Geschichte zu erzäblen ist 1967 vorbei. Die tiefgreifenden methodischen Transformationen der Annales-Schule ${ }^{19}$ inspirieren Barthes zu der Feststellung, daß zeitgenössische Geschichtsschreibung das Intelligible an die Stelle des Wirklichen gerückt habe. Nicht wissen konnte er natürlich, daß im Laufe der $80 \mathrm{er}$ Jahre ein neues geschichtliches Erzählen einsetzen würde, das verkleinerte Beobachtungsfelder erforscht und so an die Stelle des großen Zusammenhangs, der sich im Kollektivsingular die Geschichte manifestiert, die Mikroperspektive der Akteure setzt.

\section{Sprache und Wirklichkeit: die Ubiquität der Sprache}

Welches Verhältnis besteht zwischen Texten und Wirklichem? Wie schaffen sie es, daß das, was sie sagen, als, wirklich“ erscheint? „Le fait n'a jamais qu'une existence linguistique (comme terme d'un discours), et cependant tout se passe comme si cette existence n'était que la ,copie' pure et simple d'une autre existence, située dans un champ extra-structural, le , réel' “ ${ }^{20} \mathrm{Da}$ Texte prinzipiell nur aus Buchstaben, Wörtern, Sätzen etc. bestehen, sind hier - die Klammer in diesem Satz sollte man nicht überlesen - auch historische Tatsachen nur sprachlich gegeben. Die zentrale Frage ist nun für Barthes, wie Texte zeigen, daß sie Tatsachen schildern und nicht Fiktionen: „Quelle est la place du ,réel ' dans la structure discursive?" (ebd.)

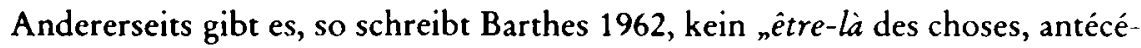
dent et extérieur au langage ${ }^{\star 21}$. Erst in mühsamer Kulturarbeit seien die Dinge von (vorgegebener stereotypisierter) Bedeutung freizumachen ( ${ }_{n}$ faire ,dé-signifier'“), wie dies z.B. der Oberflächenrealismus des Nouveau Roman anstrebe. Weil sie unmittelbar und immer bedeuten, weil sich zwischen den Wahrnehmenden und sie immer Bedeutung schiebt, und weil diese Bedeutung den direkten $\mathrm{Zu}$ gang zu den Dingen verwehrt, versuche ein Autor wie Robbe-Grillet gerade, sie als sinnfreie Oberfläche sichtbar zu machen.

Die Schwierigkeit besteht für Barthes also nicht darin, einer gegebenen Wirklichkeit Sinn zu geben und diesen auf nachvollziehbare Weise sprachlich darzustellen, sondern im Gegenteil darin, das Wirkliche von einem offenbar konstitutiven Sinnüberschuß freizumachen. Denn das Wirkliche sei immer schon von Sinn durchdrungen, mit Bedeutung überfrachtet. Grammatologisch gesprochen ist es

19 Dazu siehe Chartier (1995); Burke (1998).

20 Barthes, Le discours de l'histoire (II, 425). Da "real“ bzw. "das Reale" im Deutschen einen ziemlich materiellen, fast schon kalkulatorischen Nebensinn hat, übersetze ich "le réel“ mit "das Wirkliche“.

21 Barthes, Essais critiques I, 1322. 
,immer schon geschrieben', damit ist gemeint, es ist auf eine bestimmte Weise kodiert: "Le réel est au fond toujours déjà écrit. Il n'y a pas de réel qui ne soit déjà écrit. Tout signifiant renvoie à un autre signifiant, et ceci dans un procès infini. La réalité est toujours déjà codée, il n'y a pas de réalité qui ne soit déjà de l'écriture." 22

Wie wohl die meisten Semiologen hält Barthes semiotische Prozesse in der Wahrnehmung für allgegenwärtig. Auch, und vielleicht gerade, das nicht Sinnvolle fordere dazu auf, Sinn zu entdecken ${ }^{23}$. Anders als die meisten Semiologen sieht Barthes aber die Materialität und Sinnlichkeit der Dinge hinter ihrer Semantisierung verschwinden. Der entscheidende Faktor dieser Semantisierung ist für Barthes nun die Sprache: Durch die sprachliche Artikulation werde zwangsläufig Bedeutung produziert - gemeint ist ,zusätzliche', ,sekundäre“ Bedeutung, „Konnotation “24. Und Sprache ist überall. Sie durchdringt jede Wahrnehmung des Wirklichen ${ }^{25}$.

In dieser Hinsicht ist Barthes fest in der französischen Theorielandschaft der 60 er Jahre verankert. Seine These von der Ubiquität der Sprache ist etwa nahe an Benvenistes Feststellung, daß die Sprache in der Welt der Zeichen eine klare Vorrangstellung gegenüber anderen semiotischen Systemen habe, da sie alle Zeichen interpretieren könne, aber nicht umgekehrt ${ }^{26}$. Die spätestens mit Peirces Erkenntnistheorie allgemein akzeptierte Überzeugung, daß jede Wahrnehmung durch Zeichen vermittelt ist, spitzt Barthes also auf das Medium Sprache zu. Die Vermittlung durch Zeichen ist für ihn eine immer sprachlich unterfütterte Vermittlung: „Tout ce qui signifie dans le monde est toujours, plus ou moins, mêlé de langage: on n'a jamais de systèmes signifiants d'objets à l'état pur; le langage intervient toujours, comme relais, notamment dans les systèmes d'images, comme titres, légendes, articles. “27

22 Barthes (1971) in der Table ronde zu Exégèse et herméneutique, 249.

23 Was in der Psycholinguistik "Streben nach Sinnkonstanz" (Hörmann) heißt, ist für Barthes eine Errungenschaft von Psychoanalyse und Linguistik (I, 420). Der Ausdruck „la signification de l'insignifiance" (II, 480) deutet bereits in der Inkongruenz der Begriffsendungen -ation bzw. -ance an, daß es um unterschiedliche Sinnebenen geht, wenn in fehlenden (hewußten, intentionalen) Botschaften ein (tieferer, hintergründiger) Sinn gesucht wird. 24 Zum Konnotationsbegriff siehe Nöth (2000) $149 \mathrm{ff}$.

25 "Il n'y a de réel qu'intelligible" (Barthes, Éléments de sémiologie I [1964] 1488) und "tout système sémiologique se mêle de langage ${ }^{*}$ (I, 1412).

26 "La langue est l'interprétant de tous les systèmes sémiotiques“; „la langue peut, en principe, tout catégoriser et interpréter, y compris elle-même", Benveniste (1974) $61 \mathrm{f}$.

27 Barthes, Sémantique de l'objet II, 65. Noch deutlicher formuliert er dies in einem Gespräch von 1972: „Il n'y a aucun lieu sans langage: on ne peut pas opposer le langage, le verbal et même le verbeux à un espace pur, digne, qui serait l'espace du réel et de la vérité, un espace hors langage. Tout est langage, ou plus précisément le langage est partout. Il traverse tout le réel; il n'y a pas de réel sans langage“ (II, 1481). 


\section{Ein Realitätseffekt der Sprache}

Sprache vermittelt und strukturiert nicht nur "Wirkliches", durch eine gewisse Technik ruft sie auch den „Effekt, wirklich““ hervor, den effet de réel. Diese in der deutschen Übersetzung als „Realitätseffekt ${ }^{*}$ bekannt gewordene Wirkung der Sprache ist von Barthes sozusagen ${ }^{28}$ aus der Taufe gehoben worden. Zum ersten Mal ist von ihr im Aufsatz „Introduction à l'analyse structurale du récit“ (1966) die Rede, wo Barthes unterscheidet zwischen atmosphären Details („indices“) auf der einen Seite, deren Bedeutung (signifiés) man erst entschlüsseln müsse, und reinen Gegebenheiten, die unmittelbar bedeuten ( „données pures, immédiatement signifiantes“) auf der anderen Seite. Diese werden hier noch als Informanten („informants ${ }^{*}$ ) bezeichnet. Informanten sind zwar auch Informationsquelle für den Leser, vor allem sind sie aber Beglaubigungsstratageme von Erzählern, die bestrebt sind, ihre Geschichte im Wirklichen zu verankern. „L'informant (par exemple, l'âge précis d'un personnage) sert à authentifier la réalité du référent, à enraciner la fiction dans le réel: c'est un opérateur réaliste et, à ce titre, il possède une fonctionnalité incontestable, non au niveau de l'histoire, mais au niveau du discours.“ (II, 85)

Dazu da, in Romantexten die Realität des Referenten zu beglaubigen, rückt der ,realistische Operator', den Barthes hier noch eher en passant zum Inventar der Erzählung rechnet, im Essay „L'effet de réel“ (1968) ins Rampenlicht. Nun trägt er den Namen „détail inutile“ und wird als für die Struktur der Erzählung (l'bistoire) unbedeutende Nennung einer Einzelheit vorgestellt, die aber für das Erzählen selbst (le récit) um so bedeutungsvoller sei, weil sie wesentlich dazu beitrage, das Erzählte als wirklich (als nicht fiktiv) erscheinen zu lassen. Als Beispiel führt Barthes ein Barometer an, das am Anfang von Flauberts Erzählung „Un cour simple“ erwähnt ist: „un vieux piano supportait, sous un baromètre, un tas pyramidal de boîtes et de cartons. " 29 Weil es für die histoire keine Rolle spiele („aucune finalité ${ }^{\text {) }}$, wird es als für die Struktur der Erzählung unnütz bzw. überflüssig eingestuft (II, 479).

$\mathrm{Da}$ solchen Einordnungen immer etwas Prekäres anhaftet, liegt auf der Hand; denn das Barometer kann unter Umständen als ein Indiz atmosphärischer Milieuschilderung interpretiert oder im Kontrast zu anderen Raumbeschreibungen als signifikantes Detail gedeutet werden. Wohl auf dem Hintergrund ähnlicher Überlegungen notiert Barthes, daß durch das Zitieren die Aufmerksamkeit auf derar-

28 Zumindest dem Namen nach und was die semiotische Perspektivierung angeht - die Sache selbst, nämlich das "lebenswahre" kleine Detail, ist seit Diderots "Ëloge de Richardson" (1762) in der auch von Barthes stark gemachten Funktion der Realitätsbeglaubigung ein Thema in der Literaturtheorie; siehe den dazu bahnbrechenden Aufsatz von Hans Robert Jauß, Nachahmungsprinzip und Wirklichkeitsbegriff in der Theorie des Romans von Diderot bis Stendhal (1964).

29 Flaubert, Bd. 2 (1952) 591. 
tige Details verändert wird. Deshalb werde er seine These fortan nicht anhand von Beispielen diskutieren ${ }^{30}$.

Auf das Phänomen des absoluten Details wird Barthes in seinem Werk immer wieder zurückkommen. In „Le plaisir du texte“ (1973) führt er etwa die Schilderung eines Dîners bei Stendhal an, in dessen in den Einzelheiten benannten Bestandteilen er die Grenze sprachlicher Beschreibung ausmacht. Die detaillierte Aufzählung demonstriere, was sprachlich nicht artikulierbar sei (l'intraitable). Barthes vergleicht sie damit, daß jemand in einem Gespräch plötzlich einen Gegenstand zeigend hochhalte: „Lorsque, dans un débat, quelqu'un représente quelque chose à son interlocuteur, il ne fait qu'alléguer le demier état de la réalité, l'intraitable qui est en elle. De même, peut-être, le romancier en citant, en nommant, en notifiant la nourriture (en la traitant comme notable), impose-t-il au lecteur le dernier état de la matière, ce qui, en elle, ne peut être dépassé, reculé ... C'est cela! Ce cri ne doit pas être entendu comme une illumination de l'intelligence, mais comme la limite même de la nomination, de l'imagination.“ (II, 1517)

Die Grenze des Benennbaren wird im zeigenden Nennen also nur scheinbar überschritten. Zufällig in den Blick kommende Details, die ,nicht eigentlich von Bedeutung sind, deren Präsenz jedenfalls keinen tieferen Sinn für die Handlungsebene hat, scheinen gerade in ihrem zufälligen Genannt-Werden einen Blick auf ,den letzten Zustand der Realität' freizugeben. Die Details funktionieren nach Barthes also wie winzige, in den Text eingestreute Bilder, die immer, einen Rest Kontingenz' aufwiesen, auf den man ,mit dem Finger zeigen' könne. Worauf die Bilder zeigen, ist blanke Materie und nicht ein erst zu entschlüsselndes Signifi$\mathrm{kat}^{31}$. „Il y aurait en somme deux réalismes: le premier déchiffre le ,réel' (ce qui se démontre mais ne se voit pas); le second dit la ,réalité (ce qui se voit mais ne se démontre pas). ${ }^{32}$ Neben einer beschreibend-interpretierenden Ebene sieht Barthes damit eine nur nennende, Realität zeigende Ebene am Werk: „Le roman, qui peut mêler ces deux réalismes, ajoute à l'intelligible du, réel' la queue fantasmatique de la ,réalité': étonnement qu'on mangeât en 1791 , une salade d'oranges au rhum '...: amorce d'intelligible historique et entêtement de la chose (l'orange, le rhum) à être là." (II, 1517f.) Zwei Ebenen des Wirklichen beschäftigen Barthes in dieser Passage. Die intelligible, traditionell als wesentlicher erachtete Ebene (le réel) wäre zu dechiffrieren. Als nicht sichtbare Ebene kann man nicht auf sie zeigen. Auf die oberflächlichere réalité-Ebene hingegen kann man zeigen, indem man ihre Bestandteile aufzählt. Die aufgezählten Details, jener „phantasmatische Schwanz“ der Sprache (Orangensalat mit Rum, funktionslose Barometer über alten Pianos

\footnotetext{
30 Barthes, II, 480, Anm. 1.

31 "L'image est en effet, par nature, déictique, elle désigne, ne définit pas; il y a toujours en elle un résidu de contingence, qui ne peut être que pointé du doigt. Sémiologiquement, l'image entraîne toujours plus loin que le signifié, vers la pure matérialité du référent " (Sade, Fourier, Loyola, II, 1085).

32 Barthes spielt hier auf die Lacansche Opposition zwischen réel und réalité an, bei der sich das Wirkliche (das Reale) nachweisen lasse ( ${ }_{n}$ démontrer ${ }^{c}$ ), während man nur die Realität zeigen könne ( ${ }_{m}$ montrer ${ }^{4}$ ) (dazu ausführlicher Lindorfer [1998] 194-196).
} 
oder, wie wir gleich sehen werden, leise Klopfzeichen an kleinen Türen) manifestieren diesen Sinn für die Oberfläche. Hinter der Lust am Detail, die mitunter dazu führt, ein ganzes Abendessen bis in die Feinheiten aufzuzählen, verbirgt sich nicht nur ein perverser Hang zu zerstückelten Körpern ${ }^{33}$, sondern eben auch jener ngoût fantasmatique de la réalité" (II, 1521, H.v.m.). Fantasmatisch ist an ihm, daß er in der Fixierung auf Oberflächen und Kleinigkeiten meint, ,direkt' die ,Realität einzufangen, wo Barthes gerade zeigen will, daß eingestreute Ausdrücke wie „une salade d'oranges au rhum" oder "des confitures de Bar" nur kodierte Realitätseffekte in Gang setzen.

In der Literaturtheorie heißt das Kodiert-Sein dessen, was die histoire als das Wirkliche ausgibt, das Wabrscheinliche ${ }^{34}$. Genau dies ist es, was nach Barthes durch den Realitätseffekt entsteht: eine neue Wahrscheinlichkeit („un nouveau vraisemblable ${ }^{\star}$ ), die nun im Unterschied zu der aristotelischen oder derjenigen der französischen Klassik, wirklich“ wirken will. Der effet de réel erweist sich so als ein Kunstgriff der romanesken Illusion, den es ad acta zu legen gilt. Es gilt aufzudecken, daß die Realität, die die ,realistische' Erzählung entstehen läßt, im Verweis auf kulturelle Codes nur Wahrscheinlichkeit beanspruchen kann: „ce qu'on appelle ,réel' (dans la théorie du texte réaliste) n'est jamais qu'un code de représentation (de signification) ${ }^{\alpha 35}$.

Dieser opérateur réaliste findet sich jedoch keineswegs nur in Romanen des 19. Jahrhunderts, auch Psychoanalytiker machen z. B. von ihm Gebrauch, wenn sie sich fragen, ob Kindheitserinnerungen ,echt' sind oder nur nachträglich konstruiert. Auch ihnen garantieren unbedeutende Details die "Echtheit" einer Erinnerung. Gerade wenn das Erinnerte Züge enthält, die "nicht auflösbar" sind und die ,auch zu den aus der Phantasie stammenden Bedeutungen nicht passen “, notiert Sigmund Freud 1898, spreche dies dafür, daß „keine Erinnerungsfälschun-

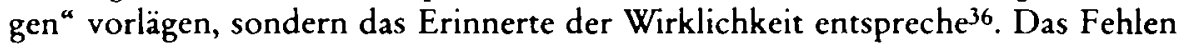
eines einsichtigen Grundes für die Präsenz eines Details indiziert in der Kontingenz, daß die Kindheitserinnerung auf wirklich Erlebtem beruht. Nicht auszuschließen ist, daß Barthes die wirklichkeitsverankernde Funktion seines détail inutile tatsächlich aus diesem Freud-Text abgeleitet hat; hier zeigt sie sich jedenfalls in ihrer Reinform.

In der Historiographie gelten natülich andere Gesetze. Hier wird weniger sinniert über scheinbar unnütze Details. Schließlich kann alles, auch die scheinbar nutzloseste Angabe, irgendwann von Nutzen sein. An einer Passage aus Michelets Revolutionsgeschichte zeigt Barthes, welche Details er im Auge hat, wenn er auch für die Geschichtsschreibung Realitätseffekte reklamiert. In diesem Passus schildert Michelet, daß Charlotte Corday kurz vor ihrer Hinrichtung von einem Maler

33 "Ce thème lacanien" (Barthes, S/Z, II, 629f.).

$34 \mathrm{Vgl}$. Todorov, Introduction zum Thema "Le vraisemblable" von Communications 11 (1968), wo auch Barthes' Essay „L'effet de réel “ zuerst erschien, und, im gleichen Heft, Genette, Vraisemblance et motivation.

35 Barthes, S/Z, II (1970) 608.

36 Freud, Über Deckerinnerungen [1898] (1977) 549. 
in ihrem Gefängnis porträtiert wurde. Michelet notiert: „Au bout d'une heure et demie, on frappa doucement à une petite porte qui était derrière elle. ${ }^{\text {"37 }}$ Barthes' Aufmerksamkeit gilt den für den Erzählablauf nicht notwendigen Angaben der Zeit, der Art und Weise des Klopfens und der Größe der Tür. Wir ahnen es: In diesen scheinbar bloß aufzählenden Kleinigkeiten steht Barthes zufolge die Authentifizierung des Erzählens im Vordergrund.

Übersetzt in die Sprache der Semiologie scheint z.B. die Kleinigkeit nau bout d'une heure et demie ${ }^{\alpha}$ unmittelbar den Sachverhalt abzubilden, Wort und Ding direkt miteinander zu verknüpfen. Der Faktor Bedeutung scheint ausgeklammert, die Wörter, bildergleich, wie die Namen der Dinge zu funktionieren. In dieser Lesart des Ausdrucks wird eine Sprachkonzeption aufgerufen, wie sie Platon im Dialog Kratylos vertreten hat und die mit Aristoteles' Interpretation der Wörter als Symbole für ihre Bedeutungen, die erst auf die Dinge verweisen ${ }^{38}$, eigentlich überwunden ist.

Natürlich scheint es nur so, daß zum Verstehen von ,unnützen' Details keine Bedeutung notwendig ist: „Dans le moment même où ces détails [inutiles] sont réputés dénoter directement le réel, ils ne font rien d'autre, sans le dire, que le signifier. " (II, 484) Auch die nutzlosesten Details vermögen tatsächlich in Texten nie zu zeigen, sondern sie bedeuten immer nur - allerdings mit der kleinen Präzision, daß ihre Sekundärbedeutung nicht auf der Ebene des Gesagten, sondern auf der des Sagens verankert ist. In einem Diskussionsbeitrag geht Barthes einmal soweit, „eine Art von Proportionalsatz“ aufzustellen: „plus le détail est insignifiant, plus il fait réel“39. Je unmotivierter die Nennung einer winzigen Kleinigkeit, desto mehr

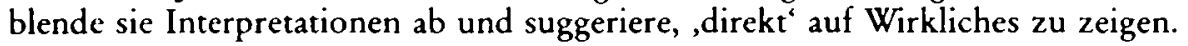
Vorausgesetzt wird in diesem Umkehrschluß übrigens eine Opposition zwischen Leben und Sinn, die Barthes selbst als ,mythisch' kennzeichnet ${ }^{40}$.

Um zusammenzufassen: Auch der Diskurs der Geschichte eliminiert (sekundäre) Bedeutungen und inszeniert eine Sprache, die in der „confusion (illusoire) du référent et du signifié ${ }^{a}$ (II, 425) eine unmittelbare Beziehung zwischen Signifikant und Referent suggeriert. Doch gerade wenn seine Darstellung den Ereignissen unmittelbar zu folgen scheint und seine Argumentation nicht erkennbar ist, werden auch hier geschichtliche Ereignisse nur bedeutet. Denn es ist ein Charakteristikum sprachlicher Zeichen, daß sie nur auf Objekte in der Wirklichkeit verweisen können, indem sie bedeuten. „Le discours historique ne suit pas le réel, il ne fait que le signifier, ne cessant de répéter c'est arrivé. " (ebd.) Als zusätzliche Bedeutung bedeutet der historiographische Text, daß von den Ereignissen in der Wirklichkeit berichtet wird. En passant erwähnte, funktionslose Zeit- und Orts-

37 Michelet, Histoire de France. La Révolution, Bd. V, 292; vgl. II, 479.

38 Aristoteles, Peri hermeneias 16a 1-10; hierzu Trabant (1996) 23-26.

39 Barthes, Exégèse et herméneutique (1971) 244.

40 Diese grande opposition mythique du vécu (du vivant) et de l'intelligible“ (II, 483) liegt nach $B$ arthes dort zugrunde, wo vermeintlich, bloße' Darstellungen des Wirklichen gegeben werden. Die Präsentation von Fakten, die so tut, als käme sie ohne eigene Deutung aus, tritt als Widerstand gegen Bedeutung auf: „comme si... ce qui vit ne pouvait signifier“ (ebd.). 
angaben oder detaillierte Aufzählung der Speisen während eines Abendessens signalisieren den Bezug eines Textes zur Wirklichkeit. Im Text selbst ist hier Faktisches und Fiktives ununterscheidbar. Er hat keinen immanenten Indikator für die Richtigkeit und Wirklichkeit seiner Angaben. Was wie ,Wirkliches 'wirkt, ist im Text selbst nur ein Effekt diskursiver Strategien.

\section{Widerspruch gegen das immer schon geschriebene Wirkliche}

Doch keineswegs herrscht diese skripturale Sicht der Dinge uneingeschränkt. In Barthes' Werk hält eine andere Stimme im Gegenteil am „être-là des choses, antécédent et extérieur au langage“ $(I, 1322)$ fest. Sie beharrt darauf, daß nicht alles immer schon von menschlichem Geist durchdrungen ist; sie besteht auf der Opposition zwischen Wirklichem und Sinn.

So betonen schon die Mythologies, daß das Wirkliche des Gegenstandes da aus dem Blick gerät, wo sein Sinn im Vordergrund steht. Das Wirkliche werde nicht nur durch stereotype Bedeutungsschübe, evakuiert' (I, 707); gerade auch die kritische Entlarvung dieser Stereotypen beschleunige seine Verflüchtigung (I, 718, Anm.). Doch weiß der ,Mythologe“ von einem Heilmittel gegen diese Agonie des Realen: die Poesie, die er als ein Sprechen unterhalb von Bedeutung („infrasignification") charakterisiert. Mit dem Ziel, „einen präsemiologischen Sprachzustand“ zu schaffen, greife die Poesie nach den Dingen selbst; sie sei deshalb letztlich nantilangage" (I, 701). Schließlich gehe sie von einem "réel finalement impénétrable, irréductible“ aus (I, 719). Um dem sich im Sprechen verflüchtigenden Wirklichen beizukommen, läßt Barthes hier allerdings noch eine zweite Methode gelten, die darin besteht „[de] poser un réel entièrement perméable à l'histoire, et idéologiser “ (ebd.). Entweder gilt es also, ausgehend von einem irreduziblen Wesen der Dinge literarisch zu schreiben oder gerade umgekehrt, die Dinge in ihrer Historizität zu durchdringen und Kulturkritik zu betreiben. In den „Mythologies“ sieht Barthes seine Aufgabe noch darin, diese beiden Alternativen miteinander zu versöhnen.

Wenn er 20 Jahre später von zwei grundsätzlich verschiedenen Annäherungsweisen an das Wirkliche spricht, hat eine tiefgreifende Transformation stattgefunden. Die Opposition besteht nicht mehr in Literatur vs. Kulturkritik, sondern in dem nun eindeutig gewichteten Gegensatzpaar système vs. systématique. Systeme setzten einen instrumentellen Sprachbegriff voraus (Illusion $n^{\circ} 1$ ) und suggerierten die wirkliche Existenz der von ihnen thematisierten Dinge (Illusion $\left.n^{\circ} 2\right)^{41}$. Das Wirkliche, auf das sich Systeme beziehen, wird als sprach- und an sich sinnlos

41 „[Le système] vit de deux illusions: une illusion de transparence (le langage dont on se sert pour l'exposer est réputé purement instrumental, ce n'est pas une écriture) et une illusion de réalité (la fin du système est qu'il soit appliqué, c'est-à-dire qu'il sorte du langage pour aller fonder un réel défini vicieusement comme l'extériorité même du langage)“ (Barthes, Sade, Fourier, Loyola, II, 1119). 
unterstellt. Anhänger des System-Denkens suchen einen Sinn zu ,dechiffrieren'. Dagegen führt Barthes das systematische Denken als ein strukturierendes ,Spiel" ein, bei dem man sich darüber im klaren ist, daß die Herangehensweise selbst zur Konstitution des Gegenstands entscheidend beiträgt. Hier wird keine absolute Distanz zu einem vermeintlich sprachfreien Gegenstand vorgespiegelt (wofür Barthes den Ausdruck ,illusion référentielle* bemüht). Barthes' Sympathie ist eindeutig auf der Seite des Systematischen. Das zeigt sich auch daran, daß das, was nun im emphatischen Sinne "écriture“ heißt, eindeutig auf dieser Seite verortet wird: „au fur et à mesure que le style s'absorbe dans l'écriture, le système se défait en systématique, le roman en romanesque, l'oraision en fantasmatique ...", schreibt er 1971 in "Sade, Fourier, Loyola“ (II, 1043) und bejubelt in "S/Z" das "Schreibbare“. "Le scriptible, c'est le romanesque sans le roman, la poésie sans le poème, l'essai sans la dissertation, l'écriture sans le style, la production sans le produit, la structuration sans la structure." (II, 558)

\section{Schreiben mit Realitätseffekt. Notieren $\&$ Zeigen}

Ausgehend von Texten entledigt Barthes sich Ende der 60er Jahre eines außersprachlichen Wirklichen mit Verweis auf strukturierende Spiele und nicht zu hintergehende écritures. Auf einem Wirklichen, das jenseits von Schrift Bestand hat, beharrt jedoch weiterhin der Schriftsteller Barthes. Er insistiert darauf, Wirkliches festhalten zu wollen.

Barthes' Beschäftigung mit dem Nouveau Roman, seine Chroniken, Reiseaufzeichungen und sein umständliches Abwägen, wie Tagebuch zu führen von künstlerischem Interesse sein kann, verbindet, daß sie die Kontingenz des Wirklichen als Herausforderung begreifen.

Eindringlich und provokativ führt der Oberflächenrealismus des Nouveau Roman neuzeitliche Kontingenzerfahrung vor Augen. Noch Mitte der 50er Jahre lautet Barthes' apodiktische Forderung, daß er genau darin zu überwinden sei: „la contingence de l'objet ne peut être en aucune façon un élément du réalisme final, car le réalisme est essentiellement signification“. Sein Statement „Nouveaux problèmes du réalisme“ verlangt „un réalisme total“, worunter eine Darstellungsweise verstanden wird, die das Kontingente der Oberfläche in der Hervorbringung ,treffender Bedeutungen“ aufhebt ( „doter de significations justes tous les paliers du réel“). Das Bedeutungslose des Zufalls, das den Objekten eigen ist, ist dazu einem Verfahren zu unterwerfen, das richtige Bedeutung generiert (oder besser vorspiegelt?). „Mais pour arriver à cela, il faut que l'écrivain s'habitue à voir la surface avec des yeux libres“ (I [1956] 551). Sinnigerweise wird Barthes in der Folge Oberflächen genauer in Augenschein nehmen. Er begibt sich auf die Suche nach vom Zufall bestimmten Ereignissen, die dennoch (oder gerade deswegen)

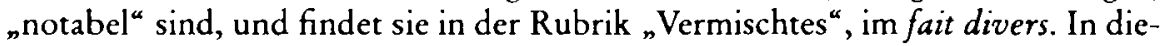
ser Textsorte ist nämlich genau das von Interesse ("l'essence même du notable ${ }^{\star}$ ), 
was auf fatale Weise von der ,fremden Macht' Zufall durchdrungen scheint (I [1962] 1313).

Doch zunehmend wird das Kontingente positiv gewendet. Es wird zum Realitätsindex schlechthin. Das Gefühl des Absurden fehlt dann völlig. Im zufälligen, nicht zu systematisierenden Detail tritt Würze (de la saveur) an die Stelle vormals beklagter Absurdität. So werden die nur durch Zufall fixierten Details der Fotografie dem Wirklichen zugeschlagen. Das zufällig fotografierte Detail demonstriert, daß das Fotografierte unwiederholbar gewesen ist, vor allem deshalb, weil es klar macht, daß es auch hätte nicht sein können. Aus diesem Grund sollen Texte nun Details notieren und Echos von Begebenheiten liefern. Wo in der ideologiekritischen Phase der fünfziger Jahre stereotype Sinngebungsmechanismen öffentlicher Gesten denunziert wurden, soll jetzt das hinter diesen Mechanismen, vergessene' Ereignis aufblitzen. Details werden aufgezählt, Dinge nur denotierend benannt, um auf wirkliche Ereignisse Bezug zu nehmen und ohne sie mit Sekundärbedeutung aufzuladen: Der Darstellungsmodus, der diesem Ideal entgegenkommt, ist das ,bloße Notieren', das scheinbar nur aufschreibt, was (zufällig) war.

$\mathrm{Ob}$ diese Aufzeichnung von Echos tatsächlich nur eine Etappe auf dem Weg, ein erster Entwurf ( ${ }_{n}$ des bouts d'essai ${ }^{*}$ ) für den eigentlichen Text ( man") ist, darüber kann man spekulieren. Für die Gegenthese, daß dieser Weg selbst das eigentliche Ziel gewesen sein könnte, spricht allerdings z.B. Barthes' letztes Buch "La chambre claire. Note sur la photographie", in dem die Spur des wirklich Gewesenen im Zentrum steht; aber auch die erst postum publizierten "Incidents" sowie haikuähnliche Anamnesen und jene "Chronique de Roland Barthes", die Ende der 70er Jahre im "Nouvel Observateur" erschien.

,Nacktes Schreiben“ („écrire à découvert“) nennt Barthes dieses Notieren was war. Es scheint keine theoretischen Voraussetzungen zu haben, schirmt sich im Gegenteil ab gegen den Sprachduktus von Semiologie, Texttheorie und Psychoanalyse. Das Material scheint auf der Straße zu liegen. Daß Chroniken solcher Ereignisse banal und leicht obszön erscheinen können, spricht allerdings manchmal dagegen, sie (zu Lebzeiten) zu publizieren.

Von Dezember 1978 bis März 1979 erschien im "Nouvel Observateur" wöchentlich die "Chronique de Roland Barthes", die nicht bedeutendes Zeitgeschehen, sondern unbedeutende Vorfälle festhielt: „Le relevé de quelques incidents qui marquent, à la semaine, ma sensibilité. ${ }^{4} 42$

Barthes' Kennzeichnung des Diskurses der geschichtlichen Chronik - „une pure série instructurée de notations ${ }^{\text {" }}$ - charakterisiert auch diese seine eigenen Texte. Wie Chronisten früherer Zeiten vermeiden auch sie sinnvollen Aufbau, Deutung und Kommentar, um die Ereignisse selbst desto stärker zur Geltung kommen zu lassen. Barthes gibt die Chronik nach drei Monaten auf. Als Ursache für das Scheitern des explizit schriftstellerischen Experiments sieht er ein prinzipielles Manko. „Le défaut, c'est qu'à chaque incident rapporté je me sens entraîné (par quelle force ou quelle faiblesse?) à lui donner un sens (social, moral, esthéti-

42 Barthes am 23. 3. 1979 im Nouvel Observateur; III, 991. 
que etc.).“, räumt er in der letzten Ausgabe vom 23. März 1979 ein (III, 992). Das Scheitern war insofern vorprogrammiert, als ja schon die „Éléments de sémiologie " verkündeten, daß das sprachlich Artikulierte unvermeidbar Bedeutung erhält.

Während das Notieren des Unbedeutenden im Wochenjournal scheitert, brilliert es als „émanation du réel“ in der Auseinandersetzung mit dem Medium Fotografie. „Toute photo est en quelque sorte co-naturelle à son référent“, notiert Barthes in „La chambre claire“ (III, 1163). Was das Licht-Bild kategorisch vom Text unterscheidet, ist sein $W_{1}$ rklichkeitsindex. Schon im Aufsatz "Le message

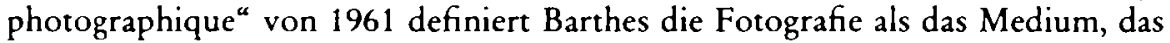
das ,buchstäblich Wirkliche' ("le réel littéral“) übermittelt, ohne dabei auf einen Code zu rekurrieren. Als ,Bild ohne Code‘ scheint die Fotografie keinerlei zusätzliche Bedeutung ins Spiel bringen zu müssen. Allerdings wird schon 1961 das vermeintlich ,perfekte Analogon" des Wirklichen, das auf den ersten Blick wie „un message sans code “ (ebd.) aussieht, als ,mythische“ Verklärung entlarvt ${ }^{43}$, indem seine konnotativen Techniken dargelegt werden. Nur für unverbesserliche ,Realisten' (wic Barthes?) ist cin fotografisches Zeichen die Sache selbst ("plus un signe mais la chose même"; III, 1138). Nichtsdestotrotz stellt die zeigende Geste der Fotografie die Herausforderung schlechthin dar für Schreiber, die das auratische Licht vergangener Ereignisse und verstorbener Personen sprachlich einfangen wollen.

Es gilt, exakt zu bezeichnen, und um dies in einer geläufigen, nicht terminologischen Sprache zu tun, ist Arbeit an der Sprache nötig. Dann entsteht das, was Barthes ,Richtigkeit' (justesse) nennt. Die Textsorte par excellence, die für Barthes in diesem Sinne ,richtig' ist, ist das japanische Haiku. Von "L'empire des signes“ an, einem Buch über die in Japan angetroffene glückliche Welt der Zeichen, ist das japanische Kurzgedicht für Barthes die Gattung, die nicht im Zeichen der Darstellung steht und doch einen Effekt in Gang setzt, der dem effet de réel ähnelt.

Pleine lune,

Et sur les nattes

L'ombre d'un pin.44

In diesem Haiku haben wir keine écriture à détails inutiles' vor uns. Aber es liefert auch keine Beschreibungen, deren Details im Hinblick auf atmosphärische oder sentimentale Konnotationen zu entschlüsseln wären. Es zielt im Gegenteil, wie diese hingeworfenen unnützen Details, darauf ab, Sekundärbedeutungen gar nicht erst aufkommen zu lassen. Doch anders als in realistischem Roman und narrativer Historiographie setzt dieses Haiku keine plakativen Signale ein, die beim Leser die zusätzliche Bedeutung, wirklich“ aufkommen lassen. „Le sens est refusé au réel; bien plus: le réel ne dispose plus du sens même du réel." (ebd.) Anzumerken bleibt, daß Barthes in seinem Kommentar des Dreizeilers nicht nur ein Wirkliches diesseits von Realitätseffekten voraussetzt, sondern auch als eines, durch

43 I, 939.

44 Zitiert in L'empire des signes, II, 803. 
dessen sprachliche Darstellung keine (,zusätzlichen') Sinnhorizonte eröffnet werden.

Das Haiku tritt an, der Sprache eine Grenze zuzuweisen ("limitation du langage"). Es geht hier darum, "d'agir sur la racine même du sens, pour obtenir que ce sens ne fuse pas, ne s'intériorise pas, ne s'implicite pas, ne se décroche pas, ne divague pas dans l'infini des métaphores, dans les sphères du symbole“ (II, 798). Was diese Negativ-Aufzählung will, ist eine Exaktheit, von der Barthes übrigens sagt, daß sie im Haiku "nullement peinture exacte du réel“ sei, sondern nadéquation du signifiant et du signifié, suppression des marges, bavures et interstices qui d'ordinaire excèdent ou ajourent le rapport sémantique" (II, 798f.). In der Übereinstimmung von Signifikant und Signifikat wird also letztlich Präzision angestrebt.

Sie soll verhindern, daß sich unendlich viele, sekundäre' Deutungen aneinanderreihen. Das unterscheidet die Präzision des Haiku nach Barthes von dem, was in der Rhetorik des Abendlandes ,kurz und bündig' heißt: „Une excellence de la concision" ist hier meist triumphales Zeichen "d'un surplus de la pensée sur le langage (la pensée l'emporte d'une longueur sur le langage) ${ }^{\alpha 5}$. Diesem Ungleichgewicht ist entgegenzuarbeiten, will man Haikus, die manchmal auch ,Anamnesen heißen ${ }^{46}$, eine ,Chronik‘ oder ,Zwischenfälle‘ notieren. Das Ideal ist, präzise auf die Dinge zu referieren.

Es gilt, bildergleich zu schreiben, Wirkliches zu zeigen. Um Interpretation und Bedeutung radikal zu beschneiden, kommen nun Elemente jenes Verfahrens neu ins Spiel, das er selbst zehn Jahre zuvor als effet de réel abgetan hatte. Seine Texte zeigen und nennen, sie operieren mit Zeichen, als wären es $\mathrm{Namen}^{47}$ oder bildhafte Textelemente. Die zeigende Darstellungsweise nähert sich dem Bild an mit dem Ziel, semiotische Prozesse auf ein Minimum zu reduzieren. Sie will nicht so sehr ausführen, wie etwas ist, sondern - gegebenenfalls durch ein hinweisendes "so!" - daß es (gewesen) ist. Auch im sprachlichen Zeigen bleibt Bedeutung (scheinbar) außen vor. ,Zeigen' heißt in der Sprache, daß ,leere' sprachliche Ausdrücke verwendet werden, die nur in gegebenen Kontexten funktionieren. Gezeigt werden kann nur auf Elemente der Situation. Die Deixis stellt einen ${ }_{n}$ Sonderfall von Referenz" dar, indem „[sich] die Beziehungen nach einem Zeigefeld [richten], in dessen ich-bier-jetzt-Origo ... der Sprecher steht ${ }^{4}{ }^{48}$. Deiktische Ele-

45 Barthes, L'ancienne rhétorique, II (1970) 936.

46 Solche ,Anamnesen' sind z. B. in der Mitte von „Roland Barthes par Roland Barthes" abgedruckt und werden in die Nähe von Haikus gerückt: "J'appelle anamnèse l'action - mélange de jouissance et d'effort - que mène le sujet pour retrouver, sans l'agrandir ni le faire vibrer, une ténuité du souvenir: c'est le haiku lui-même." (III, 178)

47 Eigennamen sind „Ausdrücke, die bestimmte Einzelobjekte.. . benennen oder bezeichnen, ohne begriffliche Information zu geben “. Ihre "primäre semantische Funktion ist ... ihre Re-

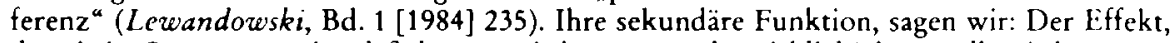
den sie in Gang setzen, ist, daß das, was sie benennen, als, wirklich' dargestellt wird.

48 Gülich, Raible (1977) 41. 
mente setzen also der Dekontextualisierung der Sprache eine Grenze, weil erst der Kontext die ,leeren' Zeichen inhaltlich füllt ${ }^{49}$.

Es gilt, Zeichen zu verwenden, die ,leer' sind bzw. die Zeichen so zu verwenden, daß ihr Sinn abgeblendet, ,ausgetrieben“ wird. „Le travail du haiku, c'est que l'exemption du sens s'accomplit à travers un discours parfaitement lisible (contradiction refusée à l'art occidental, qui ne sait contester le sens qu'en rendant son discours incompréhensible) “ (II, 802). Lesbar und doch unbedeutend (insignifiant): Das Haiku ist für Barthes die Form, die die Unlesbarkeit des Lesbaren demonstriert, ohne arrogant zu wirken. „Articulé sur une métaphysique sans sujet et sans dieu“ verkörpert es „das Erwachen vor dem Ereignis“. Einen Text wie diesen

(Je vis la première neige

Ce matin-là j'oubliai

De laver mon visage. ${ }^{50}$ )

liest Barthes als incident de langage, als sprachlichen Zwischenfall. Denn ihm gelingt, was sich das Notieren im Abendland erst erarbeiten muß: „saisie de la chose comme événement et non comme substance“ (II, 801). Das Ereignis so zu schreiben, daß noch die Lektüre zum Ereignis wird, „une cellule indissociable d'événement et d'écriture" - darin besteht die Aufgabe. Daraus die Ununterscheidbarkeit von Wirklichem und Schrift zu folgern, würde jedoch verdrängen, was seit "Écrivains et écrivants“ die „wahre Verantwortung des Schriftstellers" heißt: das Schreiben zu begreifen als ,un engagement manqué,... un regard moïséen sur la Terre Promise du réel ${ }^{\star}$ (I, 1279).

\section{Bibliographie}

Aristoteles (1982), Poetik. Griechisch/Deutsch, übersetzt u. hrsg. von Fubrmann, Manfred (Stuttgart)

Aristoteles (1994), Peri hermeneias, übersetzt von Weidemann, Hermann (Berlin)

Barberi, Alessandro (2001), Clio verwunde(r)t. Hayden White, Carlo Ginzburg und das Sprachproblem in der Geschichte (Wien)

Barthes, Roland (1971), Table ronde zu L'analyse structurale du récit: à propos d'Actes, 1011, in: Exégèse et herméneutique (Actes du congrès à Chantilly, sept. 1969) (Paris) 239-267 Barthes, Roland (1993-95), (Euvres complètes, hrsg. von Marty, Eric, 3 Bde. (Paris)

Benveniste, Émile (1991), La nature des pronoms (1956), in: Problèmes de linguistique générale I (Paris) 251-257

Benveniste, Émile (1974), Sémiologie de la langue (1969), in: Problèmes de linguistique générale II (Paris) 43-66

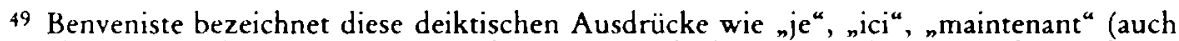
"cmbrayeurs" oder Shifter genannt) als "un ensemble de signes, vides', non référentiels par rapport à la ,réalité', toujours disponibles, et qui deviennent, pleins' dès qu'un locuteur les assume dans chaque instance de son discours“, Émile Benveniste, La nature des pronoms (1956) 254.

so Zitiert in Barthes, Empire des signes, II, 799. 
Burke, Peter (1996), Städtische Kultur in Italien zwischen Hochrenaissance und Barock. Eine historische Anthropologie (1987) (Frankfurt a.M.)

Burke, Peter (1998), Offene Geschichte. Die Schule der ,Annales' (1990) (Frankfurt a.M.)

Chartier, Roger (1995), L'Histoire Culturelle entre ,Linguistic Turn' et Retour au Sujet, in: Chartier, Roger, Vierhaus, Rudolf, Wege zu einer neuen Kulturgeschichte (Göttingen) 29 58

Daniel, Ute, Clio unter Kulturschock. Zu den aktuellen Debatten der Geschichtswissenschaft, in: Geschichte in Wissenschaft und Unterricht 48 (1997) 195-212 und 259-278

Darnton, Robert (2000), An Early Information Society: News and Media in the EighteenthCentury Paris, in: American Historical Review 105/1 (2000) 1-34

Dinges, Martin (1994), Der Maurermeister und der Finanzrichter. Ehre, Geld und soziale Kontrolle im Paris des 18. Jabrhunderts (Göttingen)

Farge, Arlette (1992), Dire et mal dire. L'opinion publique au XVIIIc siècle (Paris)

Flaubert, Gustave (1952), CEuvres, hrsg. v. Thibaudet, A. u. Dumesnil, R., Bd. 2 (Paris)

Freud, Sigmund (51977), Über Deckerinnerungen, in: Gesammelte Werke I (Frankfurt a. M.) 529-554

Genette, Gérard (1968), Vraisemblance et motivation, jetzt in: Figures II (1969) (Paris) 71-99

Ginzburg, Carlo (2002, zuerst 1979), Spurensicherung. Die Wissenschaft auf der Suche nach sich selbst (Berlin)

Gülich, Elisabeth, Raible, Wolfgang (1977), Linguistische Textmodelle. Grundlagen und Möglichkeiten (München)

Hanisch, Emst (1996), Die linguistische Wende. Geschichtswissenschaft und Literatur, in: Kulturgeschichte heute, hrsg. von Hardtwig, Wolfgang u. Webler, Hans-Ulrich (Göttingen) $212-230$

Holenstein, Pia, Schindler, Norbert (1992), Geschwätzgeschichte(n). Ein kulturhistorisches Plädoyer für die Rehabilitierung der unkontrollierten Rede, in: Dülmen, Richard van, Dynamik der Tradition (Frankfurt a.M.) 41-108

Iggers, Georg G. (1995), Zur "Linguistischen Wende" im Geschichtsdenken und in der Geschichtsschreibung, in: Geschichte und Gesellschaft 21 (1995) 557-570

Jauß, Hans Robert (1964), Nachahmungsprinzip und Wirklichkeitsbegriff in der Theorie des Romans von Diderot bis Stendhal, in: Nachahmung und Illusion, hrsg. von Jau $\beta, H . R$. (München) 157-178

Jütte, Robert (1990), Moderne Linguistik und ,Nouvelle Histoire', in: Geschichte und Gesellschaft 16 (1990) 104-120

LaCapra, Dominick (1987), Der Käse und die Würmer. Der Kosmos eines Historikers im 20. Jahrhundert, in: Geschichte und Kritik (Frankfurt a.M.) 38-64

Lafont, Cristina (1999), The Linguistic Turn in Hermeneutic Philosophy (Cambridge, Mass., London) (span.: 1993)

Lewandowski, Theodor ( $\left.{ }^{6} 1994\right)$, Linguistisches Wörterbuch, 3 Bde. (Heidelberg)

Lindorfer, Bettina (1998), Roland Barthes: Zeichen und Psychoanalyse (München)

Michelet, Jules (1967), Histoire de France. La Révolution, Bd. V (Lausanne)

Minkmar, Nils (1996), Ausgegossene Worte. Stadtbürgerlicher Ehrbegriff, Ehrenkonflikte und Habitus im Colmar des 16. Jahrhunderts in historisch-anthropologischer Perspektive (Diss. Ms)

Neubauer, Hans-Joachim (1998), Fama. Eine Geschichte des Gerüchts (Berlin)

Nöth, Winfried (2000), Handbuch zur Semiotik (Stuttgart)

Roper, Lyndal (1999), Jenseits des linguistic turn, in: Historische Anthropologie (1999) 452466

Schöttler, Peter (1997), Wer hat Angst vor dem, linguistic turn'?, in: Geschichte und Gesellschaft 23 (1997) 134-151

Simon-Muscheid, Katharina, Simon, Christian (1996), Zur Lektüre von Gerichtsquellen: Fiktionale Realität oder Alltag in Gerichtsquellen, in: Arbeit, Liebe, Streit. Texte zur Geschichte des Geschlechterverhältnisses und des Alltags. 15. bis 18. Jahrhundert, hrsg. von Rippmann, Dorothea u. a. (Liestal) 17-39 
Schwerhoff, Gerd (1999), Aktenkundig und gerichtsnotorisch. Einführung in die Historische Kriminalitätsforschung (Tübingen)

Todorov, Tvetan (1968), Introduction, in: Communications 11 (1968)

Trabant, Jürgen (1996), Elemente der Semiotik (1976) (Tübingen, Basel)

Trabant, Jürgen (2003), Sprache der Geschichte, in: Jahrbuch des Historischen Kollegs 2002 (München) 41-65

White, Hayden (1994), Metahistory. Die historische Einbildungskraft im 19. Jahrhundert in Europa (Frankfurt a.M.)

Zemon Davis, Natalie (1988), Den Kopf in der Schlinge. Gnadengesuche und ihre Erzähler (Berlin), (engl. Fiction in the Archives 1987) 
